\title{
Quantitative Determination of Solanine in Potato with Different Growing Periods in Different Areas of Ningxia
}

\author{
Danqing Zhao ${ }^{\mathrm{a}}$, Fengfeng Zhang ${ }^{\mathrm{b}}$, Yan Wuc, Qian $\mathrm{Ge}^{\mathrm{d}}$, Xiang Chen ${ }^{\mathrm{e}}$, \\ Xiaojing Wang ${ }^{\mathrm{f}, *}$
} Ningxia Research Institute of Quality Standards and Testing Technology of Agricultural Products,
Yinchuan, 750002, China

\author{
aZDQ_6264@163.com, ${ }^{\mathrm{b} 254151885 @ q q . c o m,{ }^{c} 148375897 @ q q . c o m, ~}$ \\ d278842005@qq.com, ${ }^{\text {5535358066@qq.com, }{ }^{f} 894921388 @ q q . c o m}$
}

Keywords: NingXia, Potato, solanine, Extraction, Growing period.

\begin{abstract}
The Content of solanine in potato was determined by high performance liquid chromatography (HPLC) and UV detector. The content of solanine in potato was studied in different regions and different cultivars. With the extension of the growth time, the content of solanine decreased gradually in the three growing periods of potato (tuber growth, starch accumulation and ripening). The content of solanine in the coated potato is generally higher than that of the open potato.In the growth period of tuber, the content of solanine in Long No.14 was $3.358 \mathrm{mg} / 100 \mathrm{~g}$, which was higher than that of other cultivars. With the cultivars of Open No.14, the content of solanine was lower than that of other cultivars in three growing period and the content of solanine was the lowest in mature harvest.
\end{abstract}

\section{Introduction}

Solanum is a steroid alkaloid consisting of glucose residues and solanesine, consisting essentially of $\alpha$-solanine and $\alpha$-cardosine with $\alpha$-solanine base of more than $95 \%[1]$.Solanum is a kind of secondary metabolites produced by potatoes during respiration[2].Solanum is a natural toxin, poisoning occurs so that the consumption of germinated potatoes, immature tomatoes also contain the material. When the potato tuber of solanine content of $10-15 \mathrm{mg} / 100 \mathrm{~g}$, the consumption of significant bitter taste, the content of more than $20 \mathrm{mg} / 100 \mathrm{~g}$, the food after the risk of poisoning or disease [3-6].

With the implementation of the national potato staple food and industrial development strategy, the proportion of potato staple products in the total consumption of potato has increased significantly [7].At present, Ningxia potato staple food special varieties, low level of cultivation, deep processing technology backward, single processing of processed products, staple food product development and industrial development is lagging behind and other factors constrained Ningxia potato staple food industry and staple food product development process.In this study, we used ethanol-acetic acid double solvent extraction [8] to determine the content of solanine in different cultivated areas, different cultivation patterns and different varieties of potato in Ningxia. The effects of solanine on the growth of potato tubers, starch accumulation and ripening [9] accumulation, for the potato quality and nutritional identification evaluation to make some basic research.

\section{Materials and Methods}

\subsection{Materials.}

Potato, produced in Ningxia Guyuan Touying, Xiji, Longde region, collected varieties were Long No.14, Qing No. 10, Zhong No.20.

Methanol, anhydrous ethanol, acetonitrile are chromatographic pure, acetic acid, KH2PO3 are all analytical pure, $\alpha$-solanine standard products (American sigma company).

High performance liquid chromatograph (U.S. Waters) - UV detector (2487); T18basic high speed homogenizer (Germany); Accuracy $0.01 \mathrm{mg}$ electronic balance (Swiss mettler-tolido group); UV- 
2000 ultraviolet spectrophotometer (Unico instrument co., LTD); Td-401 centrifuge (Shanghai anting scientific instrument factory); HF-5B ultrasonic circulation extractor (Ningbo Xinzhi biotechnology company); MS3 vortex mixer (German Digital company), KQ-200VDB dual-frequency numerical control ultrasonic cleaner, b-260 rotary evaporator (Shanghai Yarong chemical instrument factory)

\subsection{Methods.}

\subsubsection{Test sample preparation.}

Take ten potatoes which were no mechanical damage, no germination green and no deformed, with four cents method will be divided into 4 samples of potato samples, diagonal samples 1 cut into small pieces, high-speed homogenizer sample, stored in $-20^{\circ} \mathrm{C}$ in the refrigerator to be measured.

\subsubsection{Preparation of standard samples.}

$\alpha$-solanine is the standard reference substance for solanine. The standard was accurately weighed $10 \mathrm{mg}$ and dissolved in methanol, which was dissolved in $10 \mathrm{~mL}$ volumetric flask, prepared into 1000 $\mathrm{mg} / \mathrm{kg}$ standard mother liquor, stored in $-20{ }^{\circ} \mathrm{C}$ refrigerator.

\subsubsection{Standard curve drawing.}

Take the standard solution 5.0,10,25,50,100,200,500,1000,2000 $\mu \mathrm{L}$, placed in $10 \mathrm{~mL}$ volumetric flask respectively, and added methanol to the solution volume of $10 \mathrm{~mL}$. After mixing well, the standard solution into the liquid phase vialto be tested.The standard working curve is plotted against the concentration of the peak area.

\subsubsection{Extraction Method of Solanum nigrum in Potato.}

Accurate weighing of potato samples $10.00 \mathrm{~g}$, adding $20.00 \mathrm{~mL}$ ethanol - acetic acid (100:30, V/ V) reagent on the extraction of solanine, ultrasonic assisted extraction. Extract the centrifuge, take the supernatant $10 \mathrm{~mL}$, vacuum distillation, recovery solvent. The $\mathrm{SPE} \mathrm{NH}_{2}$ column, methanol / dichloromethane $(5: 95)$ elution, vacuum distillation, recovery reagent to the whole dry, $5 \mathrm{~mL}$ methanol volume, over $0.22 \mu \mathrm{m}$ film, to be tested.

2.2.5Determination of the content of solanine in different growth patterns and different varieties of potato during growing period.

The potato varieties were harvested in the Longde area by using the two kinds of growth methods, including the Long No.14, the Qing No.10 and the Zhong No.20, respectively.Thesolanine contents of the potato in different varieties and different growth modes were analyzed.The three growth periods of potato were tuber growth, amylum accumulation and maturity

\subsubsection{The content of solanine in different regions and different varieties of potato in growth} period was determined.

Collected samples of coated potato in Touying, Xiji and Longde of Ningxia.Collection of potato varieties were Long No.14, the Qing No.10 and the Zhong No.20.The changes of the content of longanin in three growth stages were analyzed

\section{Results and discussion}

\subsection{Method for determination of solanine in potato.}

The method for determination of solanine in potato as shown in Figure 1.The relationship between the concentration of $\alpha$-solanine $(\mathrm{x})$ and the peak area $(\mathrm{y})$ is $\mathrm{y}=20239 \mathrm{x}+10781, \mathrm{R}^{2}=0.9997$.The linear range of peak area and concentration of solanine was $0.01-200 \mathrm{mg} / 100 \mathrm{~g}$. 


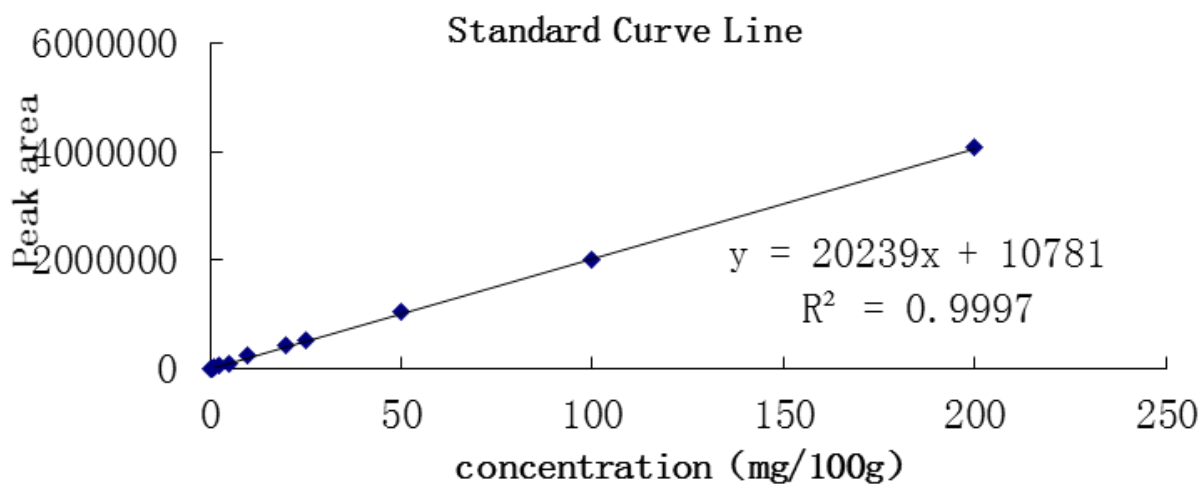

Fig. 1Standard curve of solanine in potato

The $\alpha$-solanine $10,20,50 \mu \mathrm{L}$ standard solution were added to the sample.Calculate the spike recovery rate.As shown in Table 1, the average recoveries were $82.0 \%$ and $\mathrm{RSD}$ was $1.78 \%(\mathrm{n}=3)$. The recovery of this method is in accordance with the requirements of $80 \%-120 \%$ recovery.This method can be used to determine the content of solanine in potatoes.

Table 1. Results of percent recovery tests

\begin{tabular}{cccccc}
\hline No. & $\begin{array}{c}\text { Solanine Essential } \\
\text { Additions }(\mathrm{mg} / 100 \mathrm{~g})\end{array}$ & $\begin{array}{c}\text { Detection rate of } \\
\text { solanine } \\
(\mathrm{mg} / 100 \mathrm{~g})\end{array}$ & $\begin{array}{c}\text { Recovery rate } \\
(\%)\end{array}$ & $\begin{array}{c}\text { Average recovery } \\
\text { rate }(\%)\end{array}$ & $\begin{array}{c}\text { RSD } \\
(\%)\end{array}$ \\
\hline 1 & 1.0 & 0.816 & 81.6 & & \\
2 & 2.0 & 1.69 & 84.5 & 82.9 & 1.78 \\
3 & 5.0 & 4.13 & 82.6 & & \\
\hline
\end{tabular}

\subsection{Analysis of Solanum nigrum Content in Different Growth Patterns of Different Potato} Varieties.

Growing potatoes in two ways to the coated and open.Determination of Solanum nigrum Content in Potato Planted in Longde region.As shown in Figure 2, during the three growing periods of potato, tuber growth period, starch accumulation and maturation stage, the content of solanine decreased gradually with the prolongation of time, and the content of solanine in the coated potato was generally higher than that of open potato.The content of solanine for Coated Long No. 14 in the three growing periods was higher than that of other cultivars. At the same time, in the open field conditions, Long No.14 in the content of solanine were lower than other varieties. With film conditions, the lowest content of mature solanine was Zhong No.20.The content of solanine was $0.021 \mathrm{mg} / 100 \mathrm{~g}$. This data can be used as a different planting method, the choice of different varieties to plant .

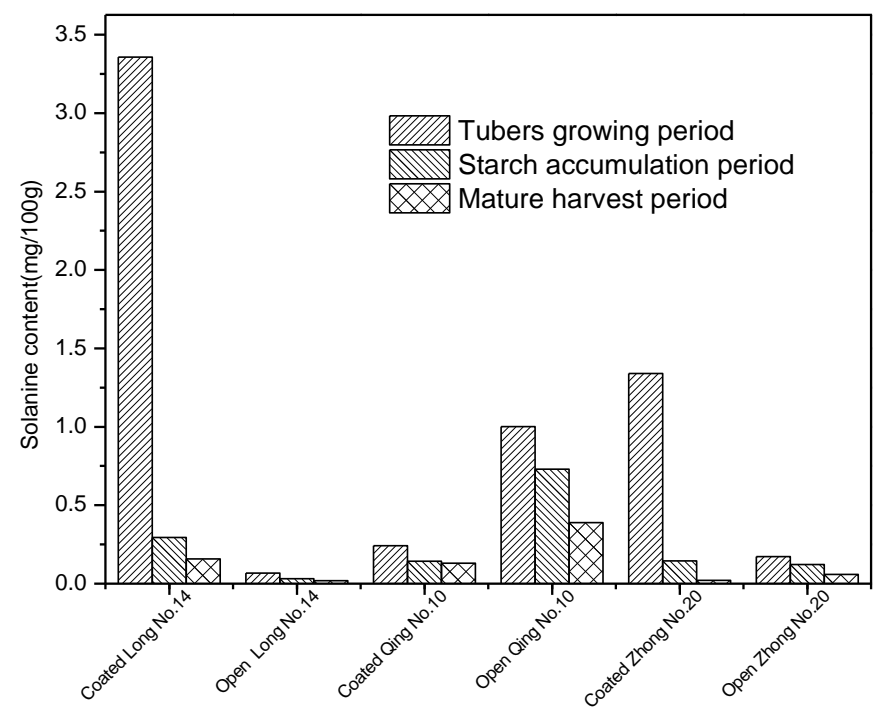

Fig. 2Analysis of Solanum Content in Different Growth Patterns of Different Potato Varieties 


\subsection{Analysis on the Content of Solanum nigrum in Potato at Different Growth Stages from Different Producing Areas and Different Growth Patterns.}

Collected in Ningxia Touying, Xiji, Longde region of the coated and open field of two kinds of growth patterns potato samples.Different origin, different growth patterns of potatoes in different growth period of solanine content was show as table 2.In the tubers growing period, the content of Coated Long No.14solaninewas higher than that of other varieties. The content of potato solanine in $\mathrm{Xiji}$ area was generally higher than that of other producing areas. This may be because the Xiji region is more arid and the higher temperature is favorable for the generation of solanine.The content of solanine was lower in the ripening period of potato cultivation in Touying area. The Touying camp could be used as the key area for potato planting.Zhong No.20 had lower solanine content in mature harvesting period under both growth conditions.Zhong No.20 can be used as a major cultivar in the Touying and Pengyang area.The lower content of solanine is an important part of measuring potato quality.

Table 2. Different origin, different growth patterns of potatoes in different growth period of solanine content

\begin{tabular}{ccccc}
\hline mg/100g & & Tubers growing period & Starch accumulation period & Mature harvest period \\
\hline \multirow{3}{*}{ Coated Long No.14 } & Longde & 3.358 & 0.294 & 0.158 \\
& Touying & 5.439 & 0.124 & 0.034 \\
& Xiji & 7.007 & 0.509 & 0.341 \\
\hline \multirow{3}{*}{ Open Long No.14 } & Longde & 0.068 & 0.032 & 0.018 \\
& Touying & 1.021 & 0.691 & 0.112 \\
& Xiji & 3.452 & 0.862 & 0.012 \\
\hline \multirow{2}{*}{ Coated Qing No.10 } & Longde & 0.241 & 0.142 & 0.131 \\
& Touying & 0.674 & 0.087 & 0.049 \\
& Xiji & 1.586 & 0.096 & 0.064 \\
\hline \multirow{3}{*}{ Open Qing No.10 } & Longde & 1.001 & 0.730 & 0.390 \\
& Touying & 1.393 & 0.318 & 0.110 \\
& Xiji & 1.344 & 0.541 & 0.123 \\
\hline \multirow{2}{*}{ Coated Zhong No.20 } & Longde & 1.341 & 0.145 & 0.021 \\
& Touying & 0.058 & 0.039 & 0.036 \\
\hline \multirow{2}{*}{ Open Zhong No.20 } & Xiji & 1.267 & 0.722 & 0.299 \\
& Longde & 0.172 & 0.123 & 0.018 \\
\hline
\end{tabular}

\section{Conclusion}

The content of solanine in potato was determined by high performance liquid chromatography (HPLC) and ultraviolet detection. The linear range of peak area and concentration of solanine was $0.01-200 \mathrm{mg} / 100 \mathrm{~g}$. The recovery rate of the method meets the experimental requirements. The growth period of tubers, starch accumulation and maturation period were three periods of potato growth. With time, solanine content decreased. The content of solanine in the coated potato was generally higher than that of the open potato.Under the condition of film mulching, the content of solanine in Zhong No.20 was low, and the content of solanine in Long No.14 was the lowest in open field. That could be used as planting reference. Touying can be used as a major area for potato cultivation.

\section{Acknowledgements}

This work was supported by grants from Science and technology innovation pilot funds of NinxiaAcademy of Agriculture and Forestry Sciences(NKYZ-16-01-05) and (NKYQ-16-06).

\section{References}

[1]. Fankui Zeng, Tianhong Zhou, Xianxue Kang,et. Al. Determination of Glycoalkaloids in Potato Tuber by HPLC.Chinese Potato Journal.Vol.29 (2015) No.5, p.263-268. 
[2]. Hongping Huang, Huachun Guo, Qiong Wang,et al.Determination of the Content of Solanine in Potato Tuber in Yunnan .Scientia Agricultura Sinica.Vol.44(2011) No.7,p.1512-1518.

[3]. Xin Zhou,Dangqi Lin,Rusong Yang,et al.Pupils with Solpadine poisoning epidemiology. Chinese Journal of School Health.Vol.28(2007) No.9,p.849-850

[4]. Zhiyuan Ren.Food poisoning caused by potato solanine.Disease Surveillance. Vol.23 (2008) No.5, p.267.

[5]. Guangming Duan, Xiaomei Zhou.Inhibition of glycoalkaloid of potato on human blood cholinesterase activity.Chinense Biochemical Journal.Vol.10 (1994) No.5, p.575-578.

[6]. Mendel Friedman, Gary M. McDonald .Potato glycoalkaloids: Chemistry, analysis, safety, and plant physiology. Critical Reviews in Plant Sciences. Vol.16 (1997), p. 55-132.

[7]. Yan $\mathrm{Wu}$.The Key to Potato Starch and the Technique of Planting. New rural technology.Vol.6 (2015) No.4, p. 4-6.

[8]. Tianshou Qian, Mengling Kang, Jjiangang Ling,et. al.Research of Extraction Process of Solpadine in Potato. Chinese Agricultural Science Bulletin. Vol.28 (2012) No.15, p.264-267.

[9]. Yanjie Li,Junnian Yang,Renghua Liu,et al.Dynamic and component analysis of sol nine in potato and bud stage during fermentation.Heilongjiang Animal Science And veterinary Medicine.Vol.13(2014) No.7,p.114-116. 